\title{
PERCEPTION AS SUBSTITUTE TRIAL AND ERROR
}

\author{
DONALD T. CAMPBELL
}

Northwestern University

The Darwinian concept of random variation and natural selection through differential survival provides a general model for the deterministic description of adaptive phenomena. Recently this model has seen increased application to the adaptive phenomena described by psychologists as problem solving or learning. It is the purpose of this paper to suggest the application of the model to a third level, that is to say, to the adaptive behavior demonstrated in carrying out or executing a well-learned habit. Such an application seems to necessitate the conceptualization of sensory and perceptual processes implied in the title.

The early comparative biologists and evolutionists assembled impressive evidence of the adaptive fit between organismic structure and environmental possibilities. To explain such fit, three principal alternatives were available. The first involved the detailed a priori planning of a prescient deity. The second involved appropriate or corrective structural modifications based on experience with the environment in question. But this model also involves prescience, however modest and distributed, in that the organism somehow foresees which modifications will fit better. Where the Lamarckian notion of inheritable habituation could be applied, some plausibility might be gained. But most instances of adaptive fit could not thus be explained. The third model was the Darwinian theory of natural selection. For this model, unlike the second, the modifications or variations are blind, are random, are individually nonappropriate, are not of the order of corrections. But by chance there do occur those which provide better fit, and these survive and are duplicated. While Darwinian theory of evolution has undergone considerable elaboration and modification, and while there has been disagreement as to the mechanism and the magnitude of the variations involved, his basic model of natural selection is uniformly accepted today, and stands as one of the great conceptual achievements of the 19th century. In its abstract or formal aspects, it is a model which may be applied to other adaptive processes, or other apparently teleological series of events in which modifications seem guided by outcome. ${ }^{1}$

${ }^{1}$ Recently this model has been applied to embryological morphogenesis and differentiation. While this application is yet incomplete, it is worthy of psychology's attention, inasmuch as the "organizing fields" of the experimental embryologists have provided an influential model for field theory in psychology. The problem has been to explain how genetic control is exercised to account for growth and differentiation into adult form. A tempting early model involved the notion that the genes of the germ plasm, containing the total blueprint for the adult organism, became distributed in the process of mitosis, so that each somatic cell contained only those genes appropriate to its body part. This model required that the genes collectively contain an almost incredible detail, with every phase of growth predestined at a cell-by-cell level. The model has been invalidated by the experimental facts of wound healing, regeneration, and, most dramatically, by the transplantation experiments done on amphibian embryos. These show that, up to a certain stage, transplanted cells will develop in conformity with the locality in which they are placed, rather than in terms of the locality of origin. From such experiments have come concepts of organizing fields with excitatory and inhibitory gradients. Such concepts provide useful summaries of the facts, and may be stated in mathematical form, but are un- 


\section{Selective Survival in Problem Solving OR LEARNING}

A second level of organismic fit to environment is to be found in the behavioral modifications that occur within the life span of a single organism. Thus, a rat's behavior comes more and more to "fit" the maze, to reflect the environmental possibilities. The consistency of the trend toward fit and the general goodness of fit achieved have tempted some writers to teleological explanations, or to explanations involving prescience on the part of the animal in small or large degree. Ashby (3) and Pringle (22) have explicitly proposed that the blind variation and selective survival model be applied to adaptation at the level of animal problem solving and learning. ${ }^{2}$ There are differences in

satisfactory in not specifying how the control comes about. Spiegelman (26) has suggested that the natural selection model be applied here. In simplified form, he sees each somatic cell as containing the full complement of genes. The genes are conceived of as potential initiators of self-duplicating enzymeformation processes, each gene producing a characteristic enzyme, which in turn determines a characteristic cellular structure. Which genes or enzymes dominate depends upon the character of the immediate surroundings, both within the cell and externally. The surroundings provide the setting for the selective propagation of some self-duplicating enzyme complexes at the expense of others with which they compete for energy and space. Amputation, transplantation, etc. change these surroundings, and thus the patterns of dominance. The pattern of growth is modified by changing the selecting conditions. While Spiegelman has not yet extended his model to describe fully the mechanism of embryological development, the direction of theoretical development provides a promising illustration of the application of the natural selection model to a dramatic instance of a seemingly teleological phenomenon.

2 The parallel between natural selection and trial-and-error learning seems obvious, once pointed out, and yet is an important and profound intellectual achievement. It is interesting to note that Lloyd Morgan and E. L. Thorndike, who were imbued with the Dar- their applications. Ashby attempts to start from scratch, and to imitate initially a protozoan as simple as Jennings' Stentor. Pringle starts at the level of mammalian brain function, and suggests a model in which the selective survival of neural inputs among central nervous system resonance patterns accounts for learning. The present paper is most influenced by Ashby. To quote from his introduction to Design for a Brain:

The work also in a sense develops a theory of the "natural selection" of behaviour-patterns. Just as in the species the truism that the dead cannot breed implies that there is a fundamental tendency for the successful to replace the unsuccessful, so in the nervous system does the truism that the unstable tends to destroy itself imply that there is a fundamental tendency for the stable to replace the unstable. Just as the gene pattern in its encounters with the environment tends toward ever better adaptation of the inherited form and function, so does a system of step- and part-functions tend toward ever better adaptation of learned behaviour $(3, p, v i)$.

Ashby has designed and built a "Homeostat" which achieves adaptation at the levels of "stability" and "ultrastability," as meticulously defined. Essential to this machine are uniselectors or stepping-switches designed to provide a series of random changes in certain internal circuits. This device is activated whenever a swinging magnet is deflected out of its normal center range. Such deflection may result from interferences with any part of the machine's circuitry, such interferences represent-

winian heritage and were proponents of the trial-and-error doctrine, seemed to miss it, or at least did not make it explicit. Ashby and Pringle, both participating in the intellectual ferment out of which cybernetics and information theory have developed, seem to have noted the parallel independently. It is not here intended to establish precedence for the notion, however. Young (38) also presents it, although less clearly. And Lewin (16, p. 66) and Asch (2, pp. 96-98) both raise the parallel, if only to reject its applicability, apparently independently of other sources. 
ing the impingement of the environment. The stepping-switches continue to emit the random changes as long as the swinging magnet is out of center, being stopped only when centering is again achieved, leaving the internal wiring as it is at that moment. In this sense the machine learns, or solves problems, introducing adaptive innovations into its own structure.

In terms of the rudiments of the general selective survival model, habit formation would be based upon (a) random variation of emitted behavior, $(b)$ selective survival of certain variations, and $(c)$ retention and duplication of surviving variations. In terms of conceptual traditions in psychology, this translates into a random trial-and-error learning model. Accepting the general correctness of the model may be made more palatable by noting that most contemporary learning theories contain a random trial-and-error component. While this is most obvious in theories of the Thorndike tradition, as in Hull, Skinner, and especially Guthrie, it is also true of the more Gestaltish theories of Tolman and Meier, as has been pointed out elsewhere ( 6$)$. The major unsolved problems lie in the mechanisms of selection and retention, and these problems are formidable. But for the purposes of the present paper it is the randomness that needs emphasis.

The term random is an unsatisfactory one. Contained in the folk terms "random," "chance," "haphazard," "fortuitous," and the like are a number of connotations which have been seized upon and formalized in mathematical and scientific thinking. These are essential in the selective-survival model to varying degrees, and the absence of strict randomness on some points becomes a source of confusion. Speaking of the device for changing internal circuits, Ashby says:
The values of the components ... were deliberately randomized by taking the actual numerical values from Fischer and Yates' Table of Random Numbers. Once built on to the uniselectors, the values of these parameters are determined at any moment by the positions of the uniselectors. Twentyfive positions on each of four uniselectors ... provide 390,625 combinations of parameter values $(3$, p. 96$)$.

The settings or "trials" of. the machine when disturbed approach randomness in all senses of the word. First, all possible settings are equiprobable. However, the device would still work even if this feature were considerably modified. Indeed, to refer to the evolutionary paradigm, it is known that the mutation frequencies for different genes vary widely. That responses differ greatly in likelihood of appearance is not an essential deviation from the model, as long as responses vary and continue to change in the face of distress.

Second, the settings or responses are independent of each other-the likelihood of a given response's occurring is not affected by the prior responses. This requirement can be considerably breached and Ashby's machine would still work, and indeed it seems unlikely that it holds strictly for the Homeostat as constructed. Furthermore, granted the frustration tolerance which the machine seems to have, it could still adapt if the uniselectors ran through the 390,625 possibilities in an orderly, systematic fashion. Mechanically it is probably simpler and more generally effective to construct the machine as Ashby has done, but it is not essential. Since there are among the 390,625 possibilities many with equivalent effects, or since for any given stress there are numerous potential resolutions, and since in a systematic order the adjacent settings would tend to have similar effects, the systematic approach would probably result more frequently in long 
runs of trials without a solution, even though the modal solution time might not be greater., 4

Third, random connotes that the settings or trials are uncorrelated with the stress, or the stimuli that set them off. While this can be abrogated considerably without incapacitating the mechanism, a high correlation between antecedent conditions and response obviously eliminates problem solving, discovery, or innovation. Fourth, random connotes that the occurrence of the trials individually is uncorrelated with the solution, and in particular that specific correct trials are no more likely to occur at any given point than specific incorrect ones. This requirement seems essential. Insofar as it is breached in empirical observations of trial-and-error learning, it is under conditions of a joint correlation of response and solution with the instigating conditions, and represents prior learning or other prior sources of partial information. Genetically determined response hierarchies to stimuli could in this sense reflect an evolutionary accumulation of partial knowledge about probably appropriate responses. "Blind variation" or "blind trial and error" are better phrases perhaps, but "blind" used in this sense has metaphorical implications which overlap with the main theme of this paper. The term "nonprescient" is perhaps most appropriate.

A fifth and essential connotation of

8 In the evolutionary setting of gene mutations, this aspect of randomness appears essential. If mutations tended to occur in a specific order, with the same mutation first, and if the great bulk of mutations are ineffective or lethal in results, then the possibility of species extinction without adaptive innovation becomes great.

${ }^{4}$ For the trial-and-error behavior of living organisms, there is another relevant consideration. A high degree of predictability in locomotor search behavior is a source of weakness is predator-prey relationships, as is emphasized in the theory of games. random or "blind" is the eschewing of any notion that a subsequent response is a "correction" of the preceding one, or makes use of the direction of error of the earlier ones. Such notions introduce imputations of prescience which a deterministic model must avoid, unless a specific mechanism for such prescience be introduced. The settings, trials, or responses succeed each other in a blind or random fashion, the subsequent responses being no more appropriate than the prior ones, except by chance.

\section{Adaptive Fit in the Execution of HabITs}

A third level of adaptive fit of organism to environment occurs in the execution of habits. Not only does the rat manifest adaptive fit in solving or learning the maze, but in addition it usually manifests adaptive fit every time it runs the maze. Usually it has not learned a specific series of muscle contractions which run off regardless of the environment. Rather, it runs in conformity with the location of walls and passageways. Start it off on a different heading, or on a different foot, and its behavior still fits. Shift the usable mode of locomotion from wading to swimming, and although double the number of leg movements is required, the behavior still fits (17). While not all learned responses manifest this character, it seems that most of them do (6). This adaptive fit in the execution of habits has been called molar behavior by Tolman (30) and others. It represents acts, not movements, or advertent rather than inadvertent responses in Guthrie's terminologies (10). Brunswik (5) has designated it as consisting of distal responses rather than proximal ones. It is clearly the kind of response designated for study by Skinner (24). It also has been characterized as object-consistent 
response, as opposed to body-consistent response (6). Without implying that there is agreement on how such behavior can be explained, it can be stated that this is the kind of response most frequently studied in contemporary learning theory. We may quote Spence on this, speaking for the "S-R Association-Reinforcement" point of view with which he identifies Hull and himself.

Both the stimulus and response are described at a molar, commonsense level, rather than in molecular, physiological units. Thus a response is described either in terms of the effects it produces in the environment, such as depressing the lever of a Skinner Box, or in terms of the changed spatial relations of the organism in its environment, for example, entering a blind alley, approaching the positive stimulus, and so on. No note is taken of the differences in the detailed movements or motor pattern of the activity. Thus all movements of the organism that result in the same environmental change are regarded as a single-response class $(25$, p. 247 , footnote).

In the present discussion, we shall use the term "object-consistent" for such flexibly fitting responses. In contrast, a body-consistent or muscle-consistent response, controlled only in terms of body parameters, may fit an environment, but only on the basis of memory -it runs itself off without the flexible fitting which characterizes the objectconsistent response.

From the considerations just reviewed, the present paper assumes two problems: First, it assumes that the problem of adaptive fit in the execution of habits (or in the execution of adaptive instincts for that matter) is a puzzle needing explanation, and one as vulnerable to teleological pseudo-explanation as were the problems of inherited organismic form and learning. Such a focus is not common in contemporary psychology, as the level of analysis chosen for data collection and theory avoids the problem. But the focus is shared by Guthrie, if by no others. Second, it undertakes to apply the selective survival model to such adaptive fit. This application proves difficult, and the task may seem gratuitously undertaken. Persistence seems justified by the success of the model for the other levels of adaptive fit, and by the absence of other explanatory frameworks for apparently teleological sequences of behavior. Application of the model to the object-consistent response requires that a random variation and selection process go on in carrying out each response, to account for the fit to environment which it shows. The existence of such a random trial-and-error process appears more readily if we examine first object-consistent responses on the part of organisms lacking distance receptors.

Blind object-consistent responses. Let us consider a blind person who has learned the task of sorting mixed machine parts into separate bins. The response of reaching is for the most part a body-consistent response, guided in terms of body orientation and memory. The final phase of the grasping, however, involves an observable blind, random groping, varying in extent depending upon the accuracy of the initial movement. Without the trial-and-error component, the object-consistent adaptive fit of the response would not be achieved. An example of a blind, fumbling, yet object-consistent response for a seeing person might be finding a cigarette lighter in a pocket. But even for a blind person's behavior, this random trial-and-error aspect of executing well-learned behavior may not be too apparent. In part, this is due to the fact that, in a simple stable environment, body-consistent responses may approach fit quite closely, leaving the random trial-and-error component to a minimum. Thus in walking on a wellknown level floor, the blind person may 
place each foot without hesitation, or visible trial and error. And on an uneven terrain, the random trial-and-error component may be safely limited to one dimension, and appear only as a hesitant feeling for the ground. But if we are to follow the logic of Ashby's presentation, and avoid any unexplained prescience on the part of the organism, the random trial-and-error component must be present in every object-consistent response.

Let us follow further our blind subject in his well-learned performance. He identifies the piece by a random scanning of its surface with his fingers. He places it without apparent hesitation in the correct bin, if the bins are large enough for a body-consistent response to be adequate. But if we observe him more closely, we note that he has searched out the correct bin with his left hand, and that this has given confidence and precision to the response of the right. Somehow, the random trial and error of the left hand has vicariously served for the right one. Similarly, he may in walking use a blind trial and error of cane movements to search out steps, walls, and doors, reducing the trial-and-error component in his walking.

Guided object-consistent responses. But most object-consistent responses have a smooth, accurate, guided quality which seems quite out of keeping with the prescribed random trial-and-error process. If the formal model for adaptive fit is to be retained at this level, the only resolution seems to be to locate the trial-and-error process in the function of the sensory organs. It is the burden of this paper that perception serves this function of trial-and-error exploration, substituting for the motor trial and error found in the blind object-consistent response.

It is probably easiest to accept this point of view for an organ of vicarious exploration like an insect's antenna, or the blind man's cane. The analogy of the radar screen as an aiming device is of help. The radar beams scan the sky in a blind sweep, blind in that it is not modified by any prior knowledge of the location of objects. When in this search a beam reflects from a plane, a gun is then appropriately aimed. The trial and error of a radar beam has substituted for a trial and error of expensive bullets. In a parallel way, a ship's radar vicariously explores the waterways, by a trial and error of radar beams learning the location of obstacles that might otherwise have been located by a trial and error of ship movements and collisions.

It is an easy transition from the radar model to the bat's supersonic echo location-in which sound waves emitted in all directions provide the substitute random trial-and-error process. Similarly, the lateral line organ of fishes seems to have the purpose of registering waves of water pressure change in such a fashion as to locate objects in terms of the echo of the fish's own swimming, and Pumphrey (23) has suggested the radar and echo-location analogy for this process.

The case for vision is most important, but cannot be made with the clarity and completion possible for the radar and echo-location examples, since the emitting process is missing. However, the notion of vision as a surrogate trial-and-error process seems not only required by the formal model but supported by other considerations. If in visual search the gross eye movements are not blindly searching, it is because other sources of information such as touch, memory, or hearing have been employed to narrow the range of search. Hebb (11) has assembled impressive data on the active searching movements that typically characterize the simplest seeing process, and his facts 
belie the implicit notion of the passive, fixed-focus eye implicit in both Gestalt and conditioning theories. But even without temporally extended scanning, the eye in a single glance provides spatial information which can substitute for motor trial and error, which can lead to smooth, guided, object-consistent responses.

Can this be fitted into the model? Perhaps. Ashby's Homeostat under stress presents to its environment a lot of alternatives, from which one that fits is selected. Similarly, the radar beam presents in its ever-repeated scanning sweeps multiple alternative loci for reflection. In both of these the alternatives are temporally extended. The rods and the cones of a fixed-focus eye can be regarded as the simultaneous presentation of a myriad of alternative loci for possible excitation, blindly available in that their location or availability does not anticipate the location of objects, except as this glance has been preceded by other glances and other sources of information. We could build a radar device in this manner, so that instead of one scanning beam of varying direction, it had a million simultaneously operating beam emitters and receivers, all of fixed aim. The learning capacity of Ashby's Homeostat lies in the range of settings it can try. The learning capacity of the radar lies in the range of directions in which it sends its beams. The learning capacity of the eye lies in the range of possibilities which it makes simultaneously available to selective excitation. Thus even without the emitting mechanism of radar, major portions of the model seem appropriate. Vision can be seen as providing data about the spatial environment intersubstitutable with what might be learned by blind trial and error. It is to be understood similarly in a deterministic way, with no appeals to prescience. It retains the basic epis- temology of trying a lot of things and seeing what works. Although the analogy is not complete nor elegant, let us explore its implications for some already established problems and points of view.

\section{Relation to Other Viewpoints}

Response guiding through feedback. Cybernetics has made a valuable contribution to learning theory through providing a thoroughly deterministic model for the treatment of purposive behavior. Among the learning theorists, Guthrie (10) and Mowrer (19) have explicitly recognized this role. Guthrie (10, p. 283) and Wiener (37) have applied the concept of feedback to the execution of object-consistent responses, with a solution similar to the present one, but differing in an important respect. They both accept the analogy of the automatic pilot and the steam-engine governor. Applied to the object-consistent response, this means that the response is guided by its effects, as fed back by kinesthetic and other evidences of outcome. Guthrie notes, too, the "trial-and-error components" of such cybernetic controls. Both Guthrie and Wiener emphasize the importance of visual feedback in this process. "As the cat reaches for the post either ready to bite or ready to claw, the movement is continuously corrected by vision and therefore may be executed from a small variety of stances or of distances from the post" $(10$, p. 283$)$. Yet the emphasis here is on the after-feedback of the results of the motor movement, as in the blind object-consistent responses described above.

In contrast, for the notion of perception as substitute trial and error, the automatic pilot and steam-engine governor are not adequate analogies. Instead, a distinction is drawn between these, in which the main motor output 
is guided by its own effect, and those other cybernetic devices in which the main motor output is guided by the results of a prior substitute output and feedback. Thus the radar controlled anti-aircraft missile is not guided by feedback from the projectile's location or outcome, but rather by the feedback from a prior output of electromagnetic waves. The movement of the radarguided ship is not controlled by feedback of the ship's contacts and collisions with other objects, but rather by the feedback of the contacts and collisions of the exploratory, substituting radar beam. Perception is seen as controlling guided distal responses in this same trial-in-advance way.

Lashley $(15$, p. 122) has argued against the theory of control of movement which "assumed that it is continued until stopped by sensations ... which indicate that the limb has reached the desired position." He finds the speed of some controlled actions are such as to "force the conclusion that an effector mechanism can be preset or primed to discharge at any given intensity or for a given duration in independence of any sensory controls." While he is referring specifically to kinesthetic feedback, his remarks might be generalized to include visual feedback from motor movement. A study by Hess (12) on the pecking of chicks also seems relevant. The normal accurate pecking, clearly a guided object-consistent response, is made systematically inaccurate when the chicks wear distorting lenses. No amount of experience, motor feedback, or visual feedback from motor effects seems to correct this error. Instead the instinctive accurate pecking seems rigidly guided by prior visual search. The reader can perhaps convince himself of the distinction by noting with what considerable accuracy he can guide his hands to an object by first looking, and then closing his eyes before he reaches. In making this distinction, I should not like to imply that Guthrie would disagree with it. Guthrie's comments on this problem are an important step in resolving long-standing disagreements in learning theory toward which he has persistently called attention. To quote him:

Advertent solutions transferred more readily to the post in a new position. The cat did not bite or claw the air in the old spot. The animal was more likely to look around and on seeing the post in a new position approach it and perform a guided act like biting or clawing. When escape had been inadvertent, this transfer to a new position did not take place and the cat repeatedly backed into the place where the post had been.

Advertent solutions obviously have many of the qualities that interest Tolman (and should interest others). They can be described as expectancies or as perceptions of means-end relationships. It is our belief that in associating the act of reaching with the sight of the post, tendencies to reach out may through previous practice be conditioned on vision and visual orientation and serve as maintaining stimuli for a sustained reaction to the post which has the same trial-anderror components as has the automatic pilot of the plane or steamship. Reaching out and touching is a skill with much practice behind it, and it is also a behavior mode which exhibits control. When the telephone rings we ultimately reach the instrument even if our chair is in a new spot and we must follow a course which never before has been followed. We respond to the bell by rising and by being ready to grasp the telephone, perhaps by being set to say "Hello." Seen obstacles are avoided. That avoiding seen obstacles is based on past training is evident from recent operations on children for cataract in which seen obstacles are not avoided.

In other words, association may result in acts as well as movements, and this is evident in cats as well as in men. The basic nature of the learning may be just as much an association of stimulus and response in an act that includes sustaining stimuli and cybernetic corrections as it was in Pavlov's salivary responses. The automatic pilot, the thermostat, the governor of the engine-all illustrate the fact that physical analogies are available in which by setting a control we govern the later behavior of a complicated machine. In animal behavior we have only to assume that 
the setting, which can itself be a physical response, is itself subject to associative learning (10, pp. 283-284).

Vicarious trial and error. Our discussion of substitute trial and error is reminiscent of the concept of Vicarious Trial and Error (VTE) given currency by Muenzinger (21) and Tolman (31). The usage is not the same, however. Their focus was on abbreviated motor movements that substituted for complete motor acts in reactivating memories, or as an approximation of thought, or as a symptom of consciousness, rather than a process in which the substitute trial and error serves as a source of current information about the immediate environment, in equivalence to locomotor exploration.

In Muenzinger's first presentation (20, p. 204) he spoke of a type of behavior which "appeared to us to be a vestige of actual trial and error behavior," and indeed "vestigal trial and error" might have been the better label for what he had in mind, as Dennis (9) has pointed out. Yet in Muenzinger's most full presentation of the notion (21) he felt that, although the animal abbreviated his exploration by not actually going into the maze, the process was none the less a "testing out" of alternate courses of action. Tolman had still earlier, in 1926 and $1927(28,29)$ presented a similar notion of the role of a rat's "wiggling his nose from side to side and finally choosing" or "running back and forth" in reactivating, enhancing, or catalyzing discriminations and associations already partly learned. While Tolman later adopted Muenzinger's term for this type of behavior, he seems to have paid little attention to the literal connotations of the label and ends up seeing the behavior compelled by competing orientation vectors (31). Thus VTE, even if somewhat inappropriately labeled, has a well-defined research usage and a specialized theoretical role in speculations on the phylogeny of conscious thought which is still of interest (19). These connotations are distinct from, and more complicated than, the simple concept of substitute trial and error here being offered. In VTE most literally interpreted there is a vicarious search of a vicariously (through memory) represented environment. And while the process is instigated by the visible objects of the choice point, it is not conceived as a search of them, or a learning about them. In contrast, the notion of perception as substitute trial and error refers to visual search as a guide to motor response, as a substitute for blind exploratory locomation, limited to the visually accessible environment. This process may go on in the execution of well-learned habits or in the execution of instincts.

Perceptual versus mnemonic expectancies. While Tolman's use of VTE does not duplicate the notion here presented, his concept of "perceptual expectancies" as parallel in effect but distinct from "mnemonic expectancies" does duplicate it. Under these rubrics Tolman in 1932 (e.g., 30, pp. 96-97, 117-118, 134-139) clearly makes the point that vision can provide information and behavioral guides equivalent to those obtainable through motor exploration. He seems alone among learning theorists in thus recognizing perception as a knowledge process substitutable for motor trial and error. While the point receives major attention in Purposive Behavior, it has not since been elaborated by Tolman and his students, and has been omitted in more recent summaries of that position. The present argument differs from Tolman's mainly in the emphasis upon the basic role of blind trial and selective retention in all adaptation or knowledge processes, with the resulting effort to interpret perception as a random search process. 
Disagreement as to "what is learned." In a previous review (6) attention has been called to the factual disagreement in available literature as to whether or not learned responses were characteristically object-consistent or body-consistent. In transposition experiments in which test conditions made possible the disentanglement of the two definitions, the learned responses were $o b$ ject-consistent in the majority of instances, but not in all. For example, in four experiments of Wickens (33; $34,35,36) 91$ per cent of those who showed transfer had acquired an object-consistent response, 9 per cent a muscle-consistent response. Likewise, in the Guthrie and Horton (10) experiments both types of response occurred. Guthrie (10, p. 283) speaking for his own data, suggests that where the responses learned were visually guided (advertent), they showed object consistency rather than movement consistency under transposition experiments. Where the puzzle-box release movement was "inadvertent" the response consistency was in terms of stereotyped movements. Similarly, Dennis (8) found that normal rats took a short cut when a maze wall was removed, but that blinded rats traced the same path as before. It seems likely that the inconsistencies in Wickens' data might similarly be resolvable through the degree of visual involvement in the original learned habit.

Insight vs. blind trial and error in problem solving. The orientation of this paper inevitably leads to agreement with Lloyd Morgan (18) and Thorndike (27) as to the fundamental importance of trial and error in problem solving. And it would promote this principle as a basic epistemology. But the trial and error will only be "blind" in situations where vision is of no use, or for animals without sight. Thus overt trial-and-error locomotion will not always be present for animals with distance receptors. In many instances, particularly under those conditions that Köhler (14) has employed, the trial and error is handled by visual search. In others, such as in Thorndike's problems, such search is useless, and overt trial and error is all that is available. In still other situations, in which transparent fences or walls are employed, the solutions offered by visual search will be misleading, and will postpone adequate solutions until the blindly haphazard responses characteristic of frustration take over. In these terms, the data of Köhler (14), Thorndike (27), Adams (1), and Guthrie and Horton (10) do not seem so far apart. Certainly in Köhler's original accounts, there is ample evidence of trial-anderror processes, of fortuitous rather than logically deduced solutions. And in Tolman's insight studies with rats $(30, \mathrm{pp}$. 169-170) the insight that appeared with an elevated maze did not appear with a tunnel maze which limited the rats' perspective. These observations can be extended to Hilgard's (13, pp. 335-338) concept of "provisional try and correction" which he would substitute for random trial and error. Such selected and already quite adequate provisional tries will only be possible on the basis of prior learning or current perceptual trial and error. And if the try is incorrect, the blind animal will learn nothing of the direction of the error, whether too high or too low, too right or too left, and thus cannot correct but can only try again blindrandomly. An animal with vision, however, can note not only the inadequacy of the try, but often the direction of the error, and thus can demonstrate the intelligent provisional try and correction.

In this orientation, a distinction can be made between insight or problemsolving behavior involving substitute 
trial and error in the perceptually immediate environment, and higher levels of "thought." Most instances of animal insight, or non-overt trial and error, are limited to relationships in the perceptually immediate environment. But certainly there are higher levels of ideation, in which the solution involves representations of more than perceptually present objects. It is not clear that this latter level has been demonstrated in animals.

Asch (2) has clearly and sympathetically presented the analogy between natural selection and trial-and-error learning, but nonetheless finds trialand-error learning inapplicable to human beings, at least. While the present point of view makes trial-and-error learning the basic process and the final resort, it would result in some criticisms of learning theories similar to Asch's. Trial-and-error and conditioning doctrines are most appropriate to blind organisms. These theories as developed so far neglect the role of the perceptual organs as distance receptors and sources of information about spatial relationships in the immediate environment. A single photosensitive cell could substitute for the eye as conceived in the theory of most learning experiments, or a single acoustic switch for the ear. This neglect has been justified from a "first things first" approach to the development of science, and by a scrupulous adherence to deterministic explanation. But the neglect must be eventually corrected. The radar analogy should help in expanding deterministic learning theory into the behavior of organisms with fully developed visual systems.

The role of distance receptors in locomotor automata. In the view of the present writer, psychology stands to gain much from the experimental construction of automata which attempt to imitate life. Among those scientists who have essayed this, none has had a clearer perspective on the important problems of psychology, nor has been more meticulous in the logical development of requirements and solutions than has Ashby. His has been a magnificent achievement, and one to which the present writer feels greatly indebted. Yet Homeostat does not seem to imitate life as successfully as Walter's (32) mechanical tortoise or Berkeley's (4) mechanical squirrel. Ashby's Homeostat does not locomote in the physical world. It stays put, and adjusts to the impingements of a very special environment. Nor has it the ability to recognize segments of the environment, and store unused response patterns for use when these recur. As Walter has commented, in imitating life it is more plant than animal. In contrast, the mechanical tortoise and squirrel locomote purposefully in our world of objects, using both locomotor trial and error and scan. ning photoreceptors. They appear life like to the naive observer.

In the particular physical world in which we live and in which evolution took place, objects that are impervious to locomotion in general also reflect or diffuse certain electromagnetic waves. This persistent ecological condition over the eons has made possible the development of organisms able to anticipate the presence and location of solid objects through a substitute exploration based upon the opaqueness of the object to light. In the evolutionary perspective, vision is based upon this environmental correlation, as is our recent development of radar. The austere logical environment of Homeostat lacks such fortunate coincidences. No environmental parameters are provided which are correlated to other environmental parameters so highly that search of one can substitute for trial and error in contact with the other.

Is vision, or some other highly precise distance and space receptor, a pre- 
requisite for locomotor object consistency on the part of animals or machines? No logical derivation is on hand to prove that this so. Our concept and illustrations of blind object-consistent responses argue against it. And yet organisms and machines achieving locomotor object consistency without a substitute exploratory mechanism are rarities. Blind individual animals do occur in seeing species and whole species of blind cave fish and moles exist. Yet rather than being the evolutionary predecessors of seeing forms, these represent the regressive evolution of forms that attained their complexity with vision. Certainly vision or echo location is the usual concomitant of locomotor forms of life, and a probable essential in a locomotor automaton.

\section{SUMMARY}

Selective survival among random variations is taken as a general paradigm for instances of organismic fit to environment. Darwinian theory of natural selection applies the model to the fit between the inherited characteristics of organisms and the opportunities provided by their habitats. Trial-anderror doctrines apply the model to learned fit between organismic response and environment. Ashby and Pringle have independently noted the formal parallel between evolution and learning.

Attention is called to a third level of organismic fit to environment, in the adaptive responses employed in the flexible execution of well-learned habits. For blind organisms, the trial-and-error component in the carrying out of a habit may be obvious; but for organisms with distance receptors, the smoothly guided yet flexible character of the execution of learned responses seems quite out of keeping with the random variation required by the model. An effort is made to resolve this incongruity by characterizing perceptual processes as substitute trial and error, containing a search component which takes the place of blind overt motor movements. The notion is a more primitive one than that of "vicarious trial and error." It seems relevant for the empirical inconsistencies in the problems of "what is learned" and "insight." As related to servo-mechanism models, the notion is to be clearly distinguished from the simple negativefeedback regulators, like thermostat or governor, in which the feedback comes from the outcome of the primary effector. But a suggestive parallel is available in complex servo-systems such as the radar-controlled guiding of ship or projectile, in which a blindly emitted beam is selectively reflected, and is used to substitute for a trial and error of ship movements or projectiles.

\section{REFERENCES}

1. ADAMs, D. K. Experimental studies of adaptive behavior in cats. Comp. psy. chol. Monogr., 1929, 6, No. 1.

2. Asc日, S. E. Social psychology. New York: Prentice-Hall, 1952.

3. Asmby, W. R. Design for a brain. New York: Wiley, 1952.

4. Berkeley, E. C. Light sensitive electronic beast. Radio Electronics, Dec., 1951, 23, No. 3, 46-48.

5. BRunswir, E. The conceptual framework of psychology. Chicago: Univer. of Chicago Press, 1952. (Int. Encycl. unified Sci., V. 1, No, 10.)

6. Campresi, D. T. Operational delineation of "what is learned" via the transposition experiment. Psychol. Rev., $1954,61,167-174$.

7. Camprels, D. T. Adaptive behavior from random response. Behavioral Sci., 1956, 1, $105-110$.

8. Dennis, W. The sensory control of the white rat in the maze habit. $J$. genet. Psychol., 1929, 36, 59-89.

9. Dennis, W., \& Russeli, R. W. Comments on recent studies of VTE. $J$. genet. Psychol., 1939, 54, 217-221.

10. GuTHRIE, E. R. The psychology of learning. (Rev. Ed.) New York: Harper, 1952 .

11. HЕвв, D. O. The organization of behavior. New York: Wiley, 1949. 
12. Hess, E. H. Maturation and learning in the development of pecking accuracy in chicks. Amer. Psychologist, 1953, 8, 367. (Abstract)

13. Hilgard, E. L. Theories of learning. New York: Appleton-Century-Crofts, 1948.

14. KöHLER, W. The mentality of apes. New York: Harcourt, Brace, 1925.

15. Lashley, K. S. The problem of serial order in behavior. In L. A. Jeffress (Ed.), Cerebral mechanisms in behavior. New York: Wiley, 1951. Pp. 112136.

16. LewIN, K. Field theory in the social sciences. New York: Harper, 1951.

17. Macrarlane, D. A. The role of kinesthesis in maze learning. Univer. Calif. Publ. Psychol, 1930, 4, 277-305.

18. Morgan, C. L. Introduction to comparative psychology. London: Scott, 1894.

19. Mowrer, O. H. Ego psychology, cybernetics, and learning theory. In D. $K$. Adams et al., Learning theory, personality theory, and clinical research. New York: Wiley, 1954. Pp. 81-90.

20. Muenzinger, K. F., \& Gentry, E. Tone discrimination in white rats. $J$. comp. Psychol, 1931, 12, 195-206.

21. Muenzinger, K. F. Vicarious trial and error at a point of choice. I. A general survey of its relation to learning efficiency. J. genet. Psychol,; 1938, 53, 75-86.

22. Pringle, J. W. S. On the parallel between learning and evolution. Behaviour, 1951, 3, 175-215.

23. Pumphrey, R. J. Hearing. In Symposia of the Society for Experimental Biology, IV. Physiological mechanisms in animal behavior. New York: Academic Press, 1950. Pp. 1-18.

24. SkInNer, B. F. The behavior of organisms. New York: Appleton-Century, 1938.

25. Spence, K. W. Theoretical interpretations of learning. In C. P. Stone (Ed.), Comparative psychology. (3rd Ed.) New York: Prentice-Hall, 1951, Ch. 8.

26. Spiegelman, S. Differentiation as the controlled production of unique enzymatic patterns. In Symposia of the
Society for Experimental Biology, II. Growth in relation to differentiation and morphogenesis. New York: Academic Press, 1948.

27. Thorndike, E. L. Animal intelligence: an experimental study of the associative processes in animals. Psychol. Rev. Monogr. Suppl., 1898, 2, No. 8.

28. Tolman, E. C. A behavioristic theory of ideas. Psychol. Rev., 1926, 33, 352369.

29. Tolman, E. C. A behaviorist's definition of consciousness. Psychol. Rev., 1927, 34, 433-439.

30. Tolman, E. C. Purposive behavior in animals and men. New York: Century, 1932.

31. Tolman, E. C. Prediction of vicarious trial and error by means of the schematic sowbug. Psychol. Rev., 1939, 46, 318-336.

32. Waster, W. G. The living brain. New York: Norton, 1953.

33. Wickens, D. D. The transference of conditioned excitation and conditioned inhibition from one muscle group to the antagonistic muscle group. $J$. exp. Psychol., 1938, 22, 101-123.

34. Wickens, D. D. The simultaneous transfer of conditioned excitation and conditioned inhibition. J. exp. Psychol., 1939, 24, 332-338.

35. Wickens, D. D. Studies of response generalization in conditioning. $\mathbf{I}$. Stimulus generalization during response generalization. J. exp. Psychol., 1943, 33, 221227.

36. Wickens, D. D. Stimulus identity as related to response specificity and response generalization. J. exp. Psychol., 1948, 38, 389-394.

37. WIENeR, N. Cybernetics. New York: Wiley, 1948.

38. Young, J. Z. Doubt and certainty in science. Cambridge, England: Cambridge Univer. Press, 1950.

39. Young, J. Z. Memory, heredity and information. In J. Huxley (Ed.), Evolution as a process. London: Allen \& Unwin, 1954.

(Received January 25, 1956) 